\title{
RNA is favourable for analysing EGFR mutations in malignant pleural effusion of
} lung cancer

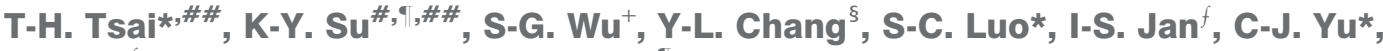 \\ S-L. Yu ${ }^{f \star * *}$, J-Y. Shih* and P-C. Yang ${ }^{*, \oplus}$
}

ABSTRACT: Malignant pleural effusion (MPE) is a useful specimen allowing for the evaluation of EGFR status in nonsmall cell lung cancer (NSCLC). However, direct sequencing of genomic DNA from MPE samples was found not to be sensitive for EGFR mutation detection.

To test whether EGFR analysis from RNA is less prone to interference from nontumour cells that have no or lower EGFR expression, we compared three methods (sequencing from cell-derived RNA versus sequencing and mass-spectrometric analysis from genomic DNA), in parallel, for EGFR mutation detection from MPE samples in 150 lung adenocarcinoma patients receiving firstline tyrosine kinase inhibitors (TKIs).

Among these MPE samples, EGFR mutations were much more frequently identified by sequencing using RNA than by sequencing and mass-spectrometric analysis from genomic DNA (for all mutations, 67.3 versus 44.7 and $46.7 \%$; for L858R or exon 19 deletions, 61.3 versus 41.3 and $46.7 \%$, respectively). The better mutation detection yield of sequencing from RNA was coupled with the superior prediction of clinical efficacy of first-line TKIs. In patients with acquired resistance, EGFR sequencing from RNA provided satisfactory detection of T790M (54.2\%).

These results demonstrated that EGFR sequencing using RNA as template greatly improves sensitivity for EGFR mutation detection from samples of MPE, highlighting RNA as the favourable source for analysing EGFR mutations from heterogeneous MPE specimens in NSCLC.

KEYWORDS: Epidermal growth factor receptor, malignant pleural effusions, nonsmall cell lung cancer, tyrosine kinase inhibitors

ung cancer, predominantly nonsmall cell lung cancer (NSCLC), is the leading cause of cancer-related death worldwide [1]. Most patients have advanced disease at the time of diagnosis and, if left untreated, have a median survival time of 4-5 months [2]. Molecular therapeutics targeting epidermal growth factor receptor (EGFR) is an appealing strategy for the treatment of advanced NSCLC [3, 4]. Recently, a strong association of somatic mutations in the tyrosine kinase domain of EGFR with clinical efficacy to two smallmolecule EGFR tyrosine kinase inhibitors (TKIs), gefitinib and erlotinib, has been clearly demonstrated [5-8]. These mutations exist in exons 18-21 of EGFR. As reported in the literature, the two major EGFR mutations, in-frame deletions in exon 19 and a single amino acid substitution at position 858 (L858R) in exon 21, were the best-documented mutations associated with response to EGFR TKIs
[6-9]. By contrast, the acquisition of a second-site EGFR mutation, T790M in exon 20, is associated with acquired TKI resistance [10, 11].

Since somatic EGFR mutations are the major determinant of tumour response to TKIs, molecular assays in clinical samples may become an integral part of care for advanced NSCLC patients [12] However, even in prospectively conducted clinical trials, $<50 \%$ of patients had specimens available for mutation analysis [13, 14]. Malignant pleural effusion (MPE) is a common complication of NSCLC. As pleural effusion sampling is usually easy, relatively noninvasive and repeatable, tumourderived DNA in MPE samples could be a useful source of information on the status of EGFR in NSCLC patients [15-18].

It is known that direct sequencing is not exquisitely sensitive in heterogeneous samples [19]. Thus, using
AFFILIATIONS

Depts of *Internal Medicine,

${ }^{\S}$ Pathology,

${ }^{f}$ Laboratory Medicine, National

Taiwan University Hospital, College of Medicine,

**Dept of Clinical Laboratory

Sciences and Medical

Biotechnology, College of Medicine,

National Taiwan University,

-Division of Genomic Medicine,

Research Center for Medical

Excellence, National Taiwan

University,

\# Institute of Statistical Science,

Academia Sinica, Taipei,

${ }^{+}$Dept of Internal Medicine, National Taiwan University Hospital Yun-Lin Branch, Yun-Lin, Taiwan.

\#\#These authors contributed equally to the study.

CORRESPONDENCE

J-Y. Shih

Dept of Internal Medicine

National Taiwan University Hospital

7 Chung-Shan South Road

Taipei 10002

Taiwan

E-mail: jyshih@ntu.edu.tw

Received:

March 092011

Accepted after revision:

June 202011

First published online:

June 302011 
sequencing of cell-derived genomic DNA from MPE samples, previous studies frequently reported an EGFR mutation rate that was lower than expected [15-18]. Interestingly, using RNA as the template for EGFR sequencing, we recently reported a much higher mutation rate in MPE samples of lung adenocarcinoma [20]. Although this variability probably reflected patient selection and geographical differences, assay methodology might have a substantial effect. Because contaminated nontumour cells within MPEs may have no or lower EGFR expression, using RNA instead of genomic DNA as the source for EGFR sequencing could minimise the influence of nontumour cells [21, 22]. However, clinical data with in-parallel comparisons will be needed to address this hypothesis and practical issues.

The sensitivity for mutation detection in heterogeneous samples may also be increased if technologies that could identify lowabundance mutations are introduced [19, 23]. Our laboratory recently established a sensitive matrix-assisted laser desorption/ ionisation time-of-flight mass spectrometry (MALDI-TOF MS) platform for DNA analysis of EGFR mutations [24, 25]. To determine whether the use of RNA may improve the sensitivity of EGFR testing, we conducted this study to compare three analytical methods (sequencing from cell-derived RNA versus sequencing and MALDI-TOF MS analysis of genomic DNA), in parallel, for the analysis of EGFR mutations from MPE samples of lung adenocarcinoma, along with the prediction of EGFR TKI efficacy in the first-line setting.

\section{METHODS}

\section{Patients and specimens}

Between June 2005 and October 2009, we consecutively collected 150 samples of MPE from 150 individual patients with advanced lung adenocarcinoma who received gefitinib or erlotinib as the first-line antitumour treatment. This study was approved by the Institutional Review Board of the National Taiwan University Hospital (Taipei, Taiwan). All patients had signed an informed

\begin{tabular}{lc} 
TABLE 1 & Clinical characteristics of patients \\
Patients n & 150 \\
Sex & \\
$\quad$ Males & $53(35.3)$ \\
$\quad$ Females & $97(64.7)$ \\
Age yrs & \\
$\quad \leqslant 65$ & $52(34.7)$ \\
$\quad>65$ & $98(65.3)$ \\
Smoking status & \\
$\quad$ Never-smokers & $113(75.3)$ \\
$\quad$ Smokers & $37(24.7)$ \\
ECOG performance status score & \\
$\quad 0-1$ & $112(74.7)$ \\
$\quad \geqslant 2$ & $38(25.3)$ \\
Clinical stage & \\
IIIb & $17(11.3)$ \\
IV & $133(88.7)$ \\
\hline
\end{tabular}

Data are presented as $n(\%)$, unless otherwise stated. ECOG: Eastern Cooperative Oncology Group. ${ }^{*}$ : based on the 6th Edn of the TNM (tumour, node and metastasis) classification of lung cancer [32]. consent form for the use of samples in molecular analysis. The adenocarcinoma histology was confirmed by the pathology reports for biopsy of the primary tumours or cell blocks of MPEs with positive thyroid transcription factor- 1 stains. Among the 150 pleural effusion samples, 94 were obtained at initial diagnosis, while the other 56 were collected with progression of the disease. Patients who had smoked $<100$ cigarettes in their lifetime were categorised as never-smokers.

\section{Extraction of genomic DNA and RNA from cell lysates of effusion samples}

The pleural effusion fluid was collected and centrifuged at $250 \times \mathrm{g}$ for $10 \mathrm{~min}$ at $4^{\circ} \mathrm{C}$, and the cell pellet was frozen. The processing of samples (from sampling to freezing) took $<2 \mathrm{~h}$. Genomic DNA was extracted from cell lysates using a QIAmp DNA Mini Kit (Qiagen, Valencia, CA, USA). For RNA purification, the cell pellet was submerged in RNAlater (Qiagen) for storage until isolation using TRI reagent (Molecular Research Center, Cincinnati, OH, USA) according to the manufacturers' instructions.

\section{Direct sequencing using cell-derived RNA}

RT-PCR was performed using a Qiagen One-Step RT-PCR Kit, with the conditions as previously described [26]. Exons 18-21 of EGFR were amplified with a forward (5"-GGA-TCG-GCC-TCTTCA-TGC-3') and reverse primer (5'-TAA-AAT-TGA-TTC-CAATGC-CAT-CC-3'). Amplicons were purified and sequenced using the BigDye Terminator Sequencing Kit (Applied Biosystems, Foster City, CA, USA). Sequencing products underwent electrophoresis on an automatic ABI PRISM 3700 genetic analyser (Applied Biosystems). Both the forward and reverse sequences obtained were analysed, and chromatograms were examined manually by two reviewers. EGFR mutations detected in the initial round of sequencing were confirmed by the subsequent round of independent RT-PCR and sequencing reactions.

\section{Direct sequencing using cell-derived genomic DNA}

Exons 18, 19, 20 and 21 of the EGFR were amplified separately by nested PCR, using specific primers (listed in table S1 in the online supplementary material) and conditions as described previously $[6,7,27-29]$. The PCR amplicons were purified and bidirectional sequencing was performed on the PCR products. Only specimens in which a mutation was confirmed in the subsequent PCR and sequencing reaction were recognised as mutation positive.

\section{MALDI-TOF MS analysis of cell-derived genomic DNA}

We performed EGFR mutation detection in genomic DNA by MALDI-TOF MS according to the users' manual of the MassARRAY system (Sequenom, San Diego, CA, USA). Briefly, after PCR of genomic DNA to amplify the loci of L858R, exon 19 deletions and T790M, single-nucleotide extension with probes was performed, followed by analysis using MALDI-TOF MS. EGFR mutants could be distinguished from wild-types due to the mass difference of an incorporated single nucleotide. For each sample, at least two replications were performed. The sequences of PCR primers and corresponding probes for identifying T790M, L858R and exon 19 deletions are listed in table S2 and illustrated in figure S1 in the online supplementary material. For exon 19 deletions, detection probes were designed for nine of the most common types of deletions. 


\section{Evaluation of EGFR TKI efficacy}

The antitumor response of the patients was evaluated by chest radiography every $2-4$ weeks and by computed tomography of the disease sites every 8-12 weeks after the start of treatment. Treatment responses were assessed according to the Response Evaluation Criteria in Solid Tumours using the unidimension method, and were reported as best response achieved [30]. Progression-free survival was calculated from the first day of TKI administration until the earliest sign of disease progression or death from any cause. Acquired resistance was defined as progression of the disease after a previously documented response or durable ( $\geqslant 6$ months) stable disease from continuous treatment with TKIs [31].

\section{Statistical analysis}

Progression-free survival after first-line TKIs was analysed by the Kaplan-Meier method, and compared between groups by the log-rank test. Analysis of factors associated with progression-free survival was performed using the Cox's proportional-hazards model. Two-sided p-values $<0.05$ were considered statistically significant. All analyses were performed using SPSS software (version 12.0 for Windows; SPSS Inc., Chicago, IL, USA).

\section{RESULTS}

\section{Patient characteristics}

The clinical characteristics of the 150 patients are summarised in table 1 . Their mean \pm SD age was $68.2 \pm 13.7$ yrs (range 30 92 yrs). Six patients received erlotinib, while the remaining 144 patients received gefitinib as the first-line anti-tumour therapy.

\section{Detection of EGFR mutations by sequencing using genomic DNA versus RNA}

All of the specimens were successfully amplified and sequenced. To determine whether the use of genomic DNA or RNA may influence the sensitivity of analysis, we compared the sequencing results using either type of template (table 2). Among the 150 MPE samples, EGFR mutations were identified in 67 (44.7\%) by sequencing of genomic DNA and in $101(67.3 \%)$ by sequencing from RNA. Of the 67 samples with mutations detected by genomic DNA sequencing, 38 (25.3\%) had L858R, 24 (16.0\%) had exon 19 deletions and five $(3.3 \%)$ had other mutations. The mutations detected by sequencing from RNA included L858R in $62(41.3 \%)$ samples, exon 19 deletions in $30(20.0 \%)$ samples and other types of mutations in nine $(6.0 \%)$ samples. Mutations other than L858R or exon 19 deletions detected by using genomic DNA or RNA are listed in table S3 in the online supplementary material.

All the mutations identified using genomic DNA were also detected using RNA, except for one sample in which the mutation (exon 19 deletion) was identified by genomic DNA sequencing only. By contrast, of the 83 samples that were identified as EGFR mutation negative by genomic DNA sequencing, 35 were found to have mutations by sequencing from RNA. Notably, analysis of the sequencing chromatograms comparing both types of template illustrated the remarkable effect of enriching mutant EGFR from tumour cells by using RNA as template (fig. 1). In the case of data revealing heterozygous mutations (double peaks), the presence of mutant allele was commonly easier to identify by using RNA, with a higher mutant/wild-type signal ratio that corresponded to the percentage of the mutant allele.

\section{Detection of L858R and exon 19 deletions by MALDI-TOF MS analysis of genomic DNA versus sequencing from RNA}

As our MALDI-TOF MS platform was designed for identifying mutations of L858R and exon 19 deletions, we focused on comparison of the mutation-detection yields between massspectrometric analysis of genomic DNA and EGFR sequencing from RNA for these two major EGFR mutations. In total, there were $70(46.7 \%)$ samples found to have L858R or exon 19 deletions by MALDI-TOF MS analysis (table 2). Our results showed that sequencing from RNA was more sensitive than mass-spectrometric analysis of genomic DNA for the detection of these two mutations. 30 samples negative for L858R or exon 19 deletions by MALDI-TOF MS analysis were identified as positive by sequencing from RNA. By contrast, sequencing from RNA missed only eight samples for the detection of these mutations, as compared with MALDI-TOF MS analysis.

Using immunocytochemical analysis (see the Methods section in the online supplementary material), we evaluated the ratio of tumour cells to total nucleated cells within MPEs in 29 effusion cell blocks, along with their influence on the yields of EGFR mutation detection by the three methods (table S4 in the online supplement material). EGFR sequencing from RNA and MALDI-TOF MS

\section{TABLE 2}

EGFR mutation status of patients detected from malignant pleural effusion samples using genomic DNA (gDNA) sequencing, sequencing from RNA or matrix-assisted laser desorption/ionisation time-of-flight mass spectrometry (MALDI-TOF MS) analysis of gDNA

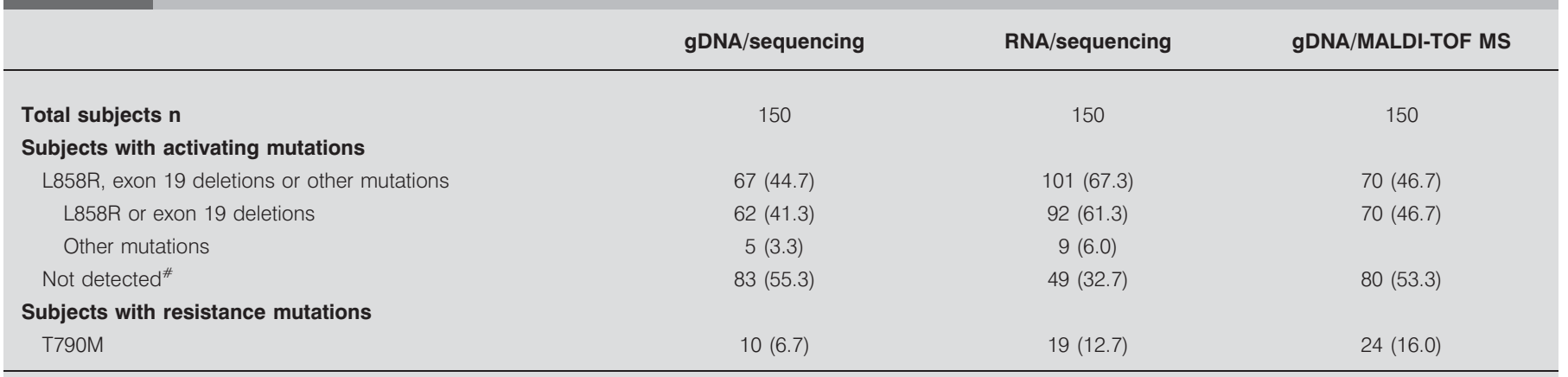

Data are presented as $n(\%)$, unless otherwise stated. * : including those with silent mutations detected by sequencing from gDNA or RNA 
a)

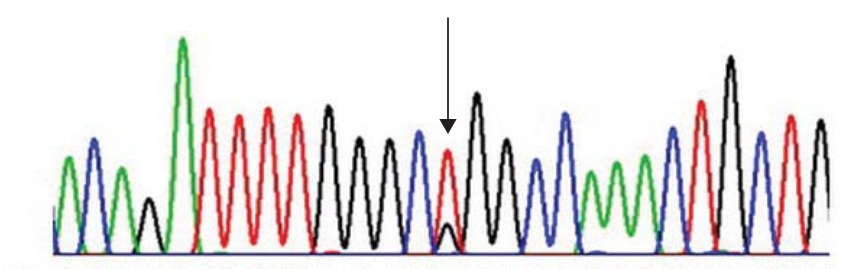

W: ACAG AT T T T G G G CT G G C CAAACT G C T G M: G

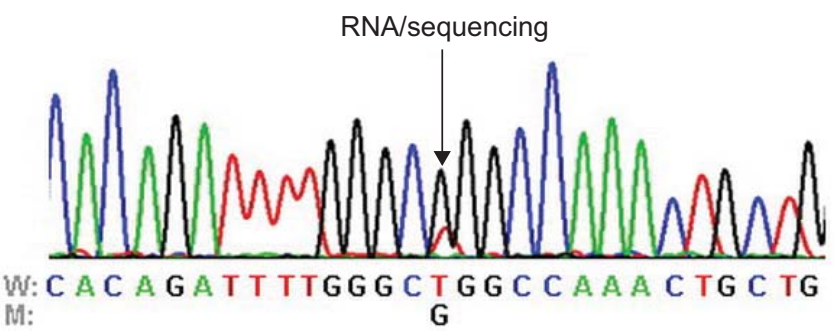

b)

gDNA/sequencing

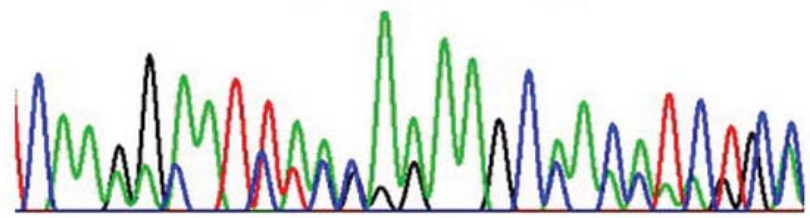

W: CAAG GATTAAGAGAAGCAACATCTC C $M: \quad A A C A C T C C G A A A G C C A A C A A G G A$ RNA/sequencing

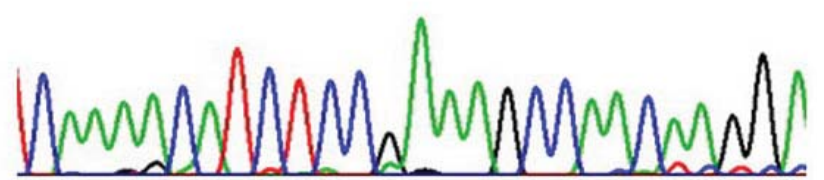

W: CAAGGAAT TAAGAGAAGCAACATCTCC M:

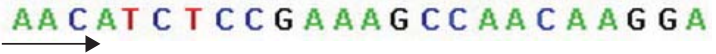

c) gDNA/sequencing

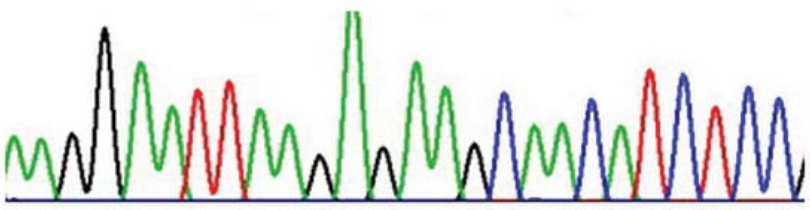

W: AAG G A A T AAGAGAAG CAACATC TC C

RNA/sequencing

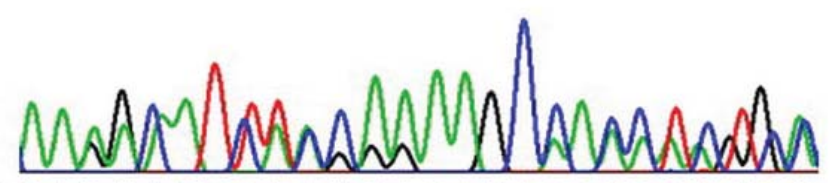

W: A A G G A T TA A G A A AG C A A CA T C TC C M: AACATCTCCGAAAGCCAACAAGGA
FIGURE 1. Representative sequencing chromatograms using genomic DNA (gDNA) or RNA as the template for EGFR sequencing. Traces are in the forward direction. The wild-type $(\mathrm{W})$ and mutant $(\mathrm{M})$ nucleotide sequences are shown below the corresponding histograms. a) Chromatograms in a patient with L858R in exon 21. The double peaks (arrows) represent the heterozygous missense mutation (2573 T>G) in the EGFR gene. Note that using RNA for EGFR sequencing, the signal of the mutant allele $(G)$ is more intense than that of the wide-type (T). b and c) Chromatograms in two samples with in-frame deletions in exon 19 (both are deletion 2235-2249). Horizontal arrows are shown to demonstrate the break point of the deletion. b) With sequencing using RNA, the peaks represent the signal of the mutant allele. The signal of wild-type allele is decreased and difficult to recognise. c) The mutant allele is not detectable by using gDNA but could be recognised by using RNA.

analysis of genomic DNA exhibited similar mutation-detection yields when tumour cells constituted $>5 \%$ of total cells within MPEs. However, the sensitivity of genomic DNA sequencing obviously decreased if tumour cells accounted for $<15 \%$ of total cells.

\section{EGFR mutation status and clinical response to first-line EGFR TKIs}

We analysed the relationship between EGFR mutation status and TKI efficacy among the 94 patients with MPE samples obtained at initial diagnosis (table 3). Of the 94 patients for analysis, partial responses occurred in $47(79.7 \%)$ out of 59 patients with identified L858R or exon 19 deletions by sequencing from RNA, and in 31 $(79.5 \%)$ out of 39 and $35(81.4 \%)$ out of 43 patients with these mutations detected by genomic DNA sequencing and MALDITOF MS analysis, respectively. Of the patients without mutations detected by genomic DNA sequencing and MALDI-TOF MS analysis, $22(42.3 \%)$ out of 52 and $18(35.3 \%)$ out of 51 patients had clinical responses. By contrast, partial responses occurred in five $(16.7 \%)$ out of 30 patients without identified mutations using sequencing from RNA. None of these five TKI responders had identified EGFR mutations by the highly sensitive Scorpion Amplified Refractory Mutation System method (EGFR RGQ PCR Kit; Qiagen) (see the Methods section in the online supplement material), suggesting the low risk of false-negative results in these patients.

Patients with L858R or exon 19 deletions had significantly longer progression-free survival than those without these mutations, according to EGFR mutation data from either genomic DNA sequencing ( $p=0.021$ by log-rank test), sequencing from RNA $(p<0.001)$ or MALDI-TOF MS analysis $(p=0.018)$ (fig. 2). Comparison of the hazard ratios (HRs) for progression $(0.64$ (95\% CI 0.41-1.00) with genomic DNA sequencing, 0.25 (95\% CI $0.15-0.41$ ) with sequencing from RNA and 0.59 (95\% CI 0.38-0.92) with TOLDI-MOF MS analysis) revealed that EGFR mutation status determined by sequencing from RNA provided the best prediction of progression-free survival (fig. 2).

\section{Detection of EGFR mutations associated with drug resistance}

Of the total 150 samples, T790M was identified in $10(6.7 \%)$ by genomic DNA sequencing, 19 (12.7\%) by sequencing from RNA and $24(16.0 \%)$ by MALDI-TOF MS analysis (table 2$)$. Among the 94 patients with samples collected at the time of initial diagnosis, de novo T790M was identified in two $(2.1 \%)$ by genomic DNA sequencing, three $(3.2 \%)$ by sequencing from RNA and eight $(8.5 \%)$ by MALDI-TOF MS analysis. Of the 56 patients with 
TABLE 3 Status of EGFR mutations assessed with different methods and clinical response to first-line epidermal growth factor receptor tyrosine kinase inhibitors in patients with malignant pleural effusion samples obtained at initial diagnosis

\begin{tabular}{|c|c|c|c|c|c|c|c|c|c|}
\hline & Subjects & \multicolumn{3}{|c|}{ gDNA/sequencing } & \multicolumn{3}{|c|}{ RNA/sequencing } & \multicolumn{2}{|c|}{ gDNA/MALDI-TOF MS } \\
\hline SD & $7(7.4)$ & $2(5.1)$ & $1(33.3)$ & $4(7.7)$ & $3(5.1)$ & $1(20.0)$ & $3(10.0)$ & $1(2.3)$ & $6(11.8)$ \\
\hline PD & $34(36.2)$ & $6(15.4)$ & $2(66.7)$ & $26(50.0)$ & $9(15.3)$ & $3(60.0)$ & $22(73.3)$ & $7(16.3)$ & 27 (52.9) \\
\hline Total $\mathbf{n}$ & 94 & 39 & 3 & 52 & 59 & 5 & 30 & 43 & 51 \\
\hline
\end{tabular}

samples obtained at the progression of disease after EGFR TKIs, we identified 24 patients fulfilling the criteria of acquired resistance to TKI treatment. Of these patients, T790M mutations were detected in six $(25.0 \%)$ by genomic DNA sequencing, 13 $(54.2 \%)$ by sequencing from RNA and $14(58.3 \%)$ by MALDI-TOF MS analysis.

\section{Factors associated with progression-free survival after treatment with first-line EGFR TKIs}

Using EGFR-mutation data determined by sequencing from RNA, we further analysed factors that were associated with progression-free survival after first-line TKI treatment among the 94 patients with MPE samples obtained at initial diagnosis (table 4). L858R or exon 19 deletions (HR 0.28, 95\% CI 0.17-0.47; $\mathrm{p}<0.001$ ) and good performance status (HR 0.58, 95\% CI 0.35 $0.97 ; p=0.036)$ were found to be independently associated with longer progression-free survival.

\section{DISCUSSION}

Molecular assays for EGFR mutations have shown promise in identifying advanced NSCLC patients who are likely to respond to EGFR TKIs. Because of the limited tissue availability for

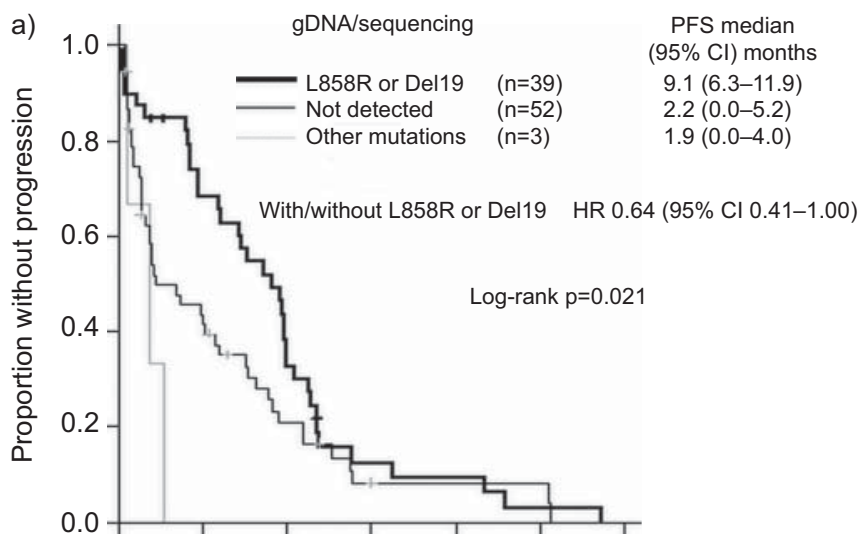

b)

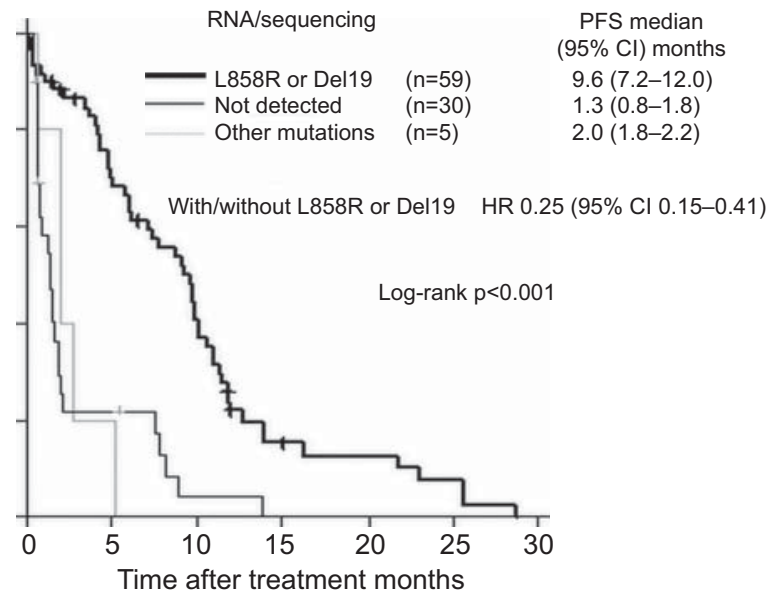

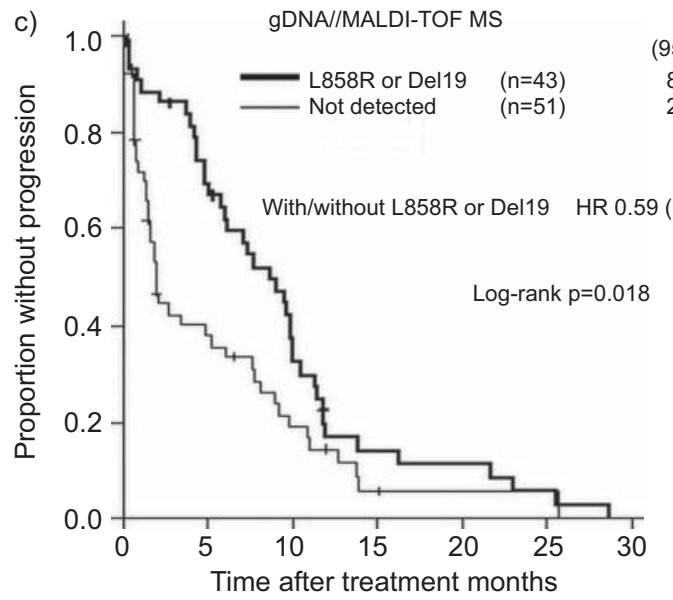

PFS median $(95 \% \mathrm{Cl})$ months $8.7(5.7-11.7)$ $2.0(1.5-2.6)$
FIGURE 2. Kaplan-Meier curves for progression-free survival (PFS) after the start of firstline epidermal growth factor receptor tyrosine kinase inhibitor treatment among patients with various statuses of EGFR mutation assessed from malignant pleural effusion samples using a) genomic DNA (gDNA) sequencing, b) sequencing from RNA and c) matrix-assisted laser desorption/ionisation time-of-flight mass spectrometry (MALDI-TOF MS) analysis of gDNA. Hazard ratios (HRs) were calculated with the use of Cox regression analysis. Del19: exon 19 deletions. 


\begin{tabular}{|c|c|c|c|}
\hline TABLE 4 & \multicolumn{3}{|c|}{$\begin{array}{l}\text { Multivariate analysis of factors associated with } \\
\text { progression-free survival to first-line epidermal } \\
\text { growth factor receptor tyrosine kinase inhibitors } \\
\text { in patients with advanced lung adenocarcinoma }\end{array}$} \\
\hline Variables & & HR $(95 \% \mathrm{Cl})$ & p-value \\
\hline \multicolumn{2}{|c|}{$\begin{array}{l}\text { L858R or exon } 19 \text { deletions, with/ } \\
\text { without mutations }\end{array}$} & $0.28(0.17-0.47)$ & $<0.001$ \\
\hline \multicolumn{2}{|c|}{ Sex, female/male } & $0.77(0.42-1.41)$ & 0.394 \\
\hline \multicolumn{2}{|c|}{$\begin{array}{l}\text { Smoking history, never/current or } \\
\text { former smokers }\end{array}$} & $0.98(0.50-1.92)$ & 0.942 \\
\hline \multicolumn{2}{|c|}{$\begin{array}{l}\text { ECOG performance status score, } \\
0-1 / \geqslant 2\end{array}$} & $0.58(0.35-0.97)$ & 0.036 \\
\hline \multicolumn{2}{|c|}{ Clinical stage $^{\#}$, IIlb/IV } & $1.04(0.54-2.01)$ & 0.901 \\
\hline \multicolumn{4}{|c|}{$\begin{array}{l}\text { HR: hazard ratio; ECOG: Eastern Cooperative Oncology Group. }{ }^{\#} \text { : based on } \\
\text { the 6th Edn of the TNM (tumour, node and metastasis) classification of lung } \\
\text { cancer [32]. }\end{array}$} \\
\hline
\end{tabular}

molecular analysis, the emerging issue concerning TKI treatment would be the development of reliable and practical EGFR testing for clinical samples that are commonly available [12, 13]. This study documented that, in contrast to analysis of genomic DNA, direct sequencing using cell-derived RNA from samples of MPE was very sensitive for EGFR mutation detection, without the complex procedures or high costs in other highly sensitive methods. This improved sensitivity was coupled with the superior prediction of treatment efficacy to first-line EGFR TKIs.

The IPASS (Iressa Pan-Asia Study), a phase 3 trial of first-line gefitinib versus chemotherapy for advanced pulmonary adenocarcinoma of never- or light smokers conducted in East Asia, reported an EGFR mutation rate of $59.7 \%$ in patients with clinical samples available for molecular analysis [14]. It is worth noting that the patients included in IPASS had favourable predictors for EGFR mutations and that a highly sensitive technique, Scorpion Amplification Refractory Mutation System, was used for EGFR testing. In the present study, using EGFR sequencing from RNA, we detected a comparable rate $(67.3 \%)$ of EGFR mutation from MPE samples. Given the high mutation-detection yield, our results suggested that EGFR sequencing using RNA from MPE samples was highly sensitive for EGFR mutation analysis in advanced lung adenocarcinoma. The parallel comparison revealed that the improved sensitivity was attributed to the use of RNA, instead of genomic DNA, as template. Thus, in contrast to direct sequencing of genomic DNA, which usually presents false-negative results, EGFR sequencing using RNA from MPE samples would be a very valuable assay for selecting advanced NSCLC patients to receive EGFR-directed therapy.

PCR-based sequencing is well established, widely available and often considered the gold standard for DNA analysis [19, 23]. The most important limitation of sequencing is the lower sensitivity for detection of somatic mutations in clinical samples when tumour DNA constitutes a small fraction of the total DNA [19]. Strategies have been developed to make mutation assays less prone to interference from nontumour cells, such as macro- or micro-dissection to enrich tumour cells before analysis [23, 33]. Based on this study, we found that the use of RNA as template is another effective approach to improve the sensitivity of EGFR testing in heterogeneous MPE samples. Compared with the frequent overexpression of EGFR in NSCLC cells, within MPEs, it is not only inflammatory cells that have no EGFR expression, as mesothelial cells also have considerably lower EGFR expression (fig. S2 in the online supplementary material) [21, 34-36]. When RNA is used as template, the differential expression of EGFR enriches the mutant EGFR of tumour cells while minimising the dilution of wild-type EGFR content from mesothelial cells (fig. S3 in the online supplementary material). This application of RNA as template offers a new dimension for EGFR analysis, especially for cytological samples, because micro-dissection is difficult to perform on cytological specimens [37]. Cytological samples, however, are more frequently used to diagnose NSCLC now, and may constitute the only available specimens in advanced-NSCLC patients who are inoperable.

Nucleotide mass spectrometry has been documented to be a sensitive assay for the analysis of oncogene mutations and genetic polymorphisms [19, 24, 38]. However, for samples of MPE, we found that MALDI-TOF MS analysis of genomic DNA was less sensitive than sequencing from RNA for EGFR mutation detection. This possibly reflected the fact that MALDI-TOF MS analysis, though more sensitive than DNA sequencing, might be still unable to circumvent the highly heterogeneous character of MPEs, in which tumour DNA might constitute an extremely small fraction of total genetic content. This observation reinforced the strength of using RNA to enrich tumour EGFR for reliable mutation testing in highly heterogeneous samples. Furthermore, compared with other mutation-specific assays (such as MALDITOF MS and others), which focus on characterised mutations, direct EGFR sequencing has the advantage of detecting rare or novel mutations as well as the known ones. Therefore, EGFR sequencing should not be disregarded in clinical use, and efforts such as using RNA instead of genomic DNA as a template should be taken to improve its performance for heterogeneous specimens.

Most studies to date have reported that $60-80 \%$ of EGFR-mutant NSCLCs respond to gefitinib or erlotinib [14, 39-42]. The present study showed a consistent response rate in patients with L858R or exon 19 deletions detected from MPEs. Using EGFR sequencing from RNA, we found that $16.7 \%$ of patients without identifiable EGFR mutations did show a partial response to TKIs. This result was equivalent to most of the literature, reporting $10-20 \%$ response rates in mutation-negative cases and indicating that EGFR mutations are not the sole determinant of TKI response [7$9,26,43,44]$. By contrast, the response rates in mutation-negative group were much higher with sequencing or MALDI-TOF MS analysis of genomic DNA as the mutation-detection method (42.3 and $35.3 \%$, respectively). Moreover, our results showed that EGFR mutation status assessed by sequencing from RNA provided the better discrimination of progression-free survival to first-line EGFR TKIs, as revealed by the HRs for progression. These findings demonstrated that EGFR sequencing using RNA from MPE samples provides a superior basis for the prediction of first-line TKI efficacy.

EGFR sequencing from RNA, though identifying more activating EGFR mutations, appears to be less sensitive for detecting T790M from MPE samples than MALDI-TOF MS analysis. One possible explanation is that, in addition to the mixing of nontumour cells in MPEs, tumour cells per se may also be 
heterogeneous, with T790M-harboring cells appearing in a small proportion of the total tumour cells, especially before treatment with EGFR TKIs [45]. While the use of RNA as template has the advantage of less dilution with EGFR from nontumour cells, the low number of de novo T790M alleles from the minority of T790M-containing tumour cells may not be readily detectable using conventional sequencing techniques. In contrast, we have shown that EGFR sequencing from RNA provided satisfactory detection of T790M (54.2\%) in patients with acquired TKI resistance. This was probably because T790M had emerged as a dominant allele through the selective pressure of TKI treatment in these patients [45].

There was one limitation of this study that needs to be noted. The timing of sampling for MPEs varied, with 94 patients sampled at the initial diagnosis and the other 56 patients at disease progression after the failure of EGFR TKIs. However, as the aim of this study was to compare different approaches in parallel for the detection of EGFR mutations from MPE samples, this limitation should not hamper our objective. Moreover, although our study suggested the promise of using RNA for EGFR analysis, the inherently labile nature of RNA, as well as the ubiquitous presence of RNase, warrants careful sample processing in RNA-based molecular testing [46].

In conclusion, we demonstrated that RNA is a more favourable source for EGFR testing than genomic DNA in the highly heterogeneous specimens of MPE related to lung cancer. Our study revealed that EGFR sequencing using RNA as template is very sensitive for mutation detection from MPE samples, which provides a basis for satisfactory prediction of treatment efficacy to first-line EGFR TKIs in advanced NSCLC.

\section{SUPPORT STATEMENT}

This study was supported by grants 98-2314-B-002-117-MY3 and 982628-B-002-087-MY3 (National Science Council, Taipei, Taiwan), and grant DOH98-TD-G-111-031 (Dept of Health, Executive Yuan, Taipei) to J-Y. Shih.

\section{STATEMENT OF INTEREST}

A statement of interest for J-Y. Shih can be found at www.erj. ersjournals.com/site/misc/statements.xhtml

\section{ACKNOWLEDGEMENTS}

The authors are grateful for the technical assistance of the Division of Genomic Medicine (Microarray Core Lab, Research Center for Medical Excellence, National Taiwan University, Taipei, Taiwan) and facility support of the Dept of Medical Research (National Taiwan University Hospital, Taipei).

\section{REFERENCES}

1 Parkin DM, Bray F, Ferlay J, et al. Global cancer statistics, 2002. CA Cancer J Clin 2005; 55: 74-108.

2 Non-small Cell Lung Cancer Collaborative Group. Chemotherapy in non-small cell lung cancer: a meta-analysis using updated data on individual patients from 52 randomised clinical trials. BMJ 1995; 311: 899-909.

3 Ciardiello F, Tortora G. EGFR antagonists in cancer treatment. N Engl J Med 2008; 358: 1160-1174.

4 Janku F, Stewart DJ, Kurzrock R. Targeted therapy in non-smallcell lung cancer - is it becoming a reality? Nat Rev Clin Oncol 2010; 7: 401-414.
5 Linardou H, Dahabreh IJ, Bafaloukos D, et al. Somatic EGFR mutations and efficacy of tyrosine kinase inhibitors in NSCLC. Nat Rev Clin Oncol 2009; 6: 352-366.

6 Paez JG, Jänne PA, Lee JC, et al. EGFR mutations in lung cancer: correlation with clinical response to gefitinib therapy. Science 2004; 304: 1497-1500.

7 Lynch TJ, Bell DW, Sordella R, et al. Activating mutations in the epidermal growth factor receptor underlying responsiveness of non-small-cell lung cancer to gefitinib. N Engl J Med 2004; 350: 2129-2139.

8 Pao W, Miller V, Zakowski M, et al. EGF receptor gene mutations are common in lung cancers from "never smokers" and are associated with sensitivity of tumours to gefitinib and erlotinib. Proc Natl Acad Sci USA 2004; 101: 13306-13311.

9 Sharma SV, Bell DW, Settleman J, et al. Epidermal growth factor receptor mutations in lung cancer. Nat Rev Cancer 2007; 7: 169-181.

10 Kobayashi S, Boggon TJ, Dayaram T, et al. EGFR mutation and resistance of non-small-cell lung cancer to gefitinib. $N$ Engl J Med 2005; 352: 786-792.

11 Pao W, Miller VA, Politi KA, et al. Acquired resistance of lung adenocarcinomas to gefitinib or erlotinib is associated with a second mutation in the EGFR kinase domain. PLoS Med 2005; 2: e73.

12 Gazdar AF. Personalized medicine and inhibition of EGFR signaling in lung cancer. N Engl J Med 2009; 361: 1018-1020.

13 Pao W, Chmielecki J. Rational, biologically based treatment of EGFR-mutant non-small-cell lung cancer. Nat Rev Cancer 2010; 10: 760-774.

14 Mok TS, Wu YL, Thongprasert S, et al. Gefitinib or carboplatinpaclitaxel in pulmonary adenocarcinoma. N Engl J Med 2009; 361: 947-957.

15 Soh J, Toyooka S, Aoe K, et al. Usefulness of EGFR mutation screening in pleural fluid to predict the clinical outcome of gefitinib treated patients with lung cancer. Int J Cancer 2006; 119: 2353-2358.

16 Kimura H, Fujiwara $Y$, Sone $T$, et al. High sensitivity detection of epidermal growth factor receptor mutations in the pleural effusion of non-small cell lung cancer patients. Cancer Sci 2006; 97: 642-648.

17 Kimura H, Fujiwara Y, Sone T, et al. EGFR mutation status in tumour-derived DNA from pleural effusion fluid is a practical basis for predicting the response to gefitinib. Br J Cancer 2006; 95: 1390-1395.

18 Hung MS, Lin CK, Leu SW, et al. Epidermal growth factor receptor mutations in cells from non-small cell lung cancer malignant pleural effusions. Chang Gung Med J 2006; 29: 373-379.

19 Pao W, Ladanyi M. Epidermal growth factor receptor mutation testing in lung cancer: searching for the ideal method. Clin Cancer Res 2007; 13: 4954-4955.

$20 \mathrm{Wu} \mathrm{SG}$, Gow $\mathrm{CH}, \mathrm{Yu} \mathrm{CJ}$, et al. Frequent epidermal growth factor receptor gene mutations in malignant pleural effusion of lung adenocarcinoma. Eur Respir J 2008; 32: 924-930.

21 GeneCards V3. EGFR gene. www.genecards.org/cgi-bin/carddisp. pl?gene $=$ EGFR\&search $=$ EGFR Date last updated: June 1, 2011. Date last accessed: June 9, 2011.

22 Gallegos Ruiz MI, Floor K, Rijmen F, et al. EGFR and K-ras mutation analysis in non-small cell lung cancer: comparison of paraffin embedded versus frozen specimens. Cell Oncol 2007; 29: 257-264.

23 Eberhard DA, Giaccone G, Johnson BE, et al. Biomarkers of response to epidermal growth factor receptor inhibitors in NonSmall-Cell Lung Cancer Working Group: standardization for use in the clinical trial setting. J Clin Oncol 2008; 26: 983-994.

24 Jurinke $C$, Oeth $P$, van den Boom D. MALDI-TOF mass spectrometry: a versatile tool for high-performance DNA analysis. Mol Biotechnol 2004; 26: 147-164. 
25 Chang TH, Tsai MF, Su KY, et al. Slug confers resistance to the epidermal growth factor receptor tyrosine kinase inhibitor. Am J Respir Crit Care Med 2011; 183: 1071-1079.

26 Mitsudomi $\mathrm{T}$, Kosaka $\mathrm{T}$, Endoh $\mathrm{H}$, et al. Mutations of the epidermal growth factor receptor gene predict prolonged survival after gefitinib treatment in patients with non-small-cell lung cancer with postoperative recurrence. J Clin Oncol 2005; 23: 2513-2520.

27 Wu SG, Chang YL, Hsu YC, et al. Good response to gefitinib in lung adenocarcinoma of complex epidermal growth factor receptor (EGFR) mutations with the classical mutation pattern. Oncologist 2008; 13: 1276-1284.

$28 \mathrm{Wu}$ JY, Yu CJ, Yang CH, et al. First- or second-line therapy with gefitinib produces equal survival in non-small cell lung cancer. $A m$ J Respir Crit Care Med 2008; 178: 847-853.

29 Shih JY, Gow CH, Yu CJ, et al. Epidermal growth factor receptor mutations in needle biopsy/aspiration samples predict response to gefitinib therapy and survival of patients with advanced nonsmall cell lung cancer. Int J Cancer 2006; 118: 963-969.

30 Eisenhauer EA, Therasse P, Bogaerts J, et al. New response evaluation criteria in solid tumours: revised RECIST guideline (version 1.1). Eur J Cancer 2009; 45: 228-247.

31 Jackman D, Pao W, Riely GJ, et al. Clinical definition of acquired resistance to epidermal growth factor receptor tyrosine kinase inhibitors in non-small-cell lung cancer. J Clin Oncol 2010; 28: 357-360.

32 Union for International Cancer Control. TNM Classification of Malignant Tumours. 6th Edn. Hoboken, John Wiley \& Sons, 2002.

33 Papadopoulos N, Kinzler KW, Vogelstein B. The role of companion diagnostics in the development and use of mutationtargeted cancer therapies. Nat Biotechnol 2006; 24: 985-995.

34 Hirsch FR, Varella-Garcia M, Bunn PA Jr, et al. Epidermal growth factor receptor in non-small-cell lung carcinomas: correlation between gene copy number and protein expression and impact on prognosis. J Clin Oncol 2003; 21: 3798-3807.

35 Suzuki S, Dobashi Y, Sakurai H, et al. Protein overexpression and gene amplification of epidermal growth factor receptor in nonsmall cell lung carcinomas. An immunohistochemical and fluorescence in situ hybridization study. Cancer 2005; 103: 1265-1273.
36 Brabender J, Danenberg KD, Metzger R, et al. Epidermal growth factor receptor and HER2-neu mRNA expression in non-small cell lung cancer is correlated with survival. Clin Cancer Res 2001; 7: 1850-1855.

37 Smouse JH, Cibas ES, Jänne PA, et al. EGFR mutations are detected comparably in cytologic and surgical pathology specimens of nonsmall cell lung cancer. Cancer Cytopathol 2009; 117: 67-72.

38 Thomas RK, Baker AC, Debiasi RM, et al. High-throughput oncogene mutation profiling in human cancer. Nat Genet 2007; 39: 347-351.

39 Morita S, Okamoto I, Kobayashi K, et al. Combined survival analysis of prospective clinical trials of gefitinib for non-small cell lung cancer with EGFR mutations. Clin Cancer Res 2009; 15: 44934498 .

40 Rosell R, Moran T, Queralt C, et al. Screening for epidermal growth factor receptor mutations in lung cancer. N Engl J Med 2009; 361: 958-967.

41 Inoue A, Kobayashi K, Usui K, et al. First-line gefitinib for patients with advanced non-small-cell lung cancer harboring epidermal growth factor receptor mutations without indication for chemotherapy. J Clin Oncol 2009; 27: 1394-1400.

42 Sequist LV, Martins RG, Spigel D, et al. First-line gefitinib in patients with advanced non-small-cell lung cancer harboring somatic EGFR mutations. J Clin Oncol 2008; 26: 2442-2449.

43 Yang CH, Yu CJ, Shih JY, et al. Specific EGFR mutations predict treatment outcome of stage IIIB/IV patients with chemotherapynaive non-small-cell lung cancer receiving first-line gefitinib monotherapy. J Clin Oncol 2008; 26: 2745-2753.

44 Taguchi F, Solomon B, Gregorc V, et al. Mass spectrometry to classify non-small-cell lung cancer patients for clinical outcome after treatment with epidermal growth factor receptor tyrosine kinase inhibitors: a multicohort cross-institutional study. J Natl Cancer Inst 2007; 99: 838-846.

45 Maheswaran S, Sequist LV, Nagrath S, et al. Detection of mutations in EGFR in circulating lung-cancer cells. N Engl J Med 2008; 359: 366-377.

46 Ladd AC, O'Sullivan-Mejia E, Lea T, et al. Preservation of fineneedle aspiration specimens for future use in RNA-based molecular testing. Cancer Cytopathol 2011; 119: 103-110. 